\section{Nanoparticles containing siRNA to silence CD4 and CCR5 reduce expression of these receptors and inhibit HIV-1 infection in human female reproductive tract tissue explants}

\author{
Susan K. Eszterhas, ${ }^{1,2}$ Nicole O. Ilonzo, ${ }^{2}$ \\ Jennifer E. Crozier,' Stela Celaj,'2 \\ Alexandra L. Howell1,2 \\ 'V.A. Medical Center, White River \\ Junction, VT; ${ }^{2}$ Department of \\ Microbiology \& Immunology, Dartmouth \\ Medical School, Lebanon, NH, USA
}

\section{Abstract}

Human Immunodeficiency Virus-type 1 (HIV1) binds to CD4 and CCR5 receptors on target cells in the human female reproductive tract. We sought to determine whether reducing levels of messenger RNA (mRNA) transcripts that encode these receptors in female reproductive tract cells could protect mucosal tissue explants from HIV1 infection. Explants prepared from the endometrium, endocervix, and ectocervix of hysterectomy tissues from HIV-1 sero-negative women were exposed to nanoparticles containing CD4- and CCR5-specific short-interfering RNA (siRNA) sequences. Explants were then exposed two days later to HIV-1, and HIV-1 reverse transcripts were measured five days post-infection. Explants treated with nanoparticles containing CD4- and CCR5-specific siRNA showed reduced levels of CD4 and CCR5 transcripts, and significantly lower levels of HIV-1 reverse transcripts compared to those treated with an irrelevant siRNA. In female reproductive tract explants and in peripheral blood cell cultures, siRNA transfection induced the secretion of IFN-alpha (IFN- $\alpha$ ), a potent antiviral cytokine. In female mice, murine-specific Cd4-siRNA nanoparticles instilled within the uterus significantly reduced murine $\mathrm{Cd} 4$ transcripts by day 3 . Our findings demonstrate that siRNA nanoparticles reduce expression of HIV-1 infectivity receptors in human female reproductive tract tissues and also inhibit HIV-1 infection. Murine studies demonstrate that nanoparticles can penetrate the reproductive tract tissues in vivo and silence gene expression. The induction of IFN- $\alpha$ after siRNA transfection can potentially contribute to the antiviral effect. These findings support the therapeutic development of nanoparticles to deliver siRNA molecules to silence host cell receptors in the female reproductive tract as a novel microbicide to inhibit mucosal HIV-1 transmission.

\section{Introduction}

Women acquire HIV-1 infection after exposure to cell free or cell-associated virus in the female reproductive tract or the rectal mucosa. Viral particles or cell-associated virus in semen infect susceptible target cells in the underlying lamina propria. ${ }^{1-3}$ The most likely targets for infection include macrophages, dendritic cells (DC), Langerhans cells and T cells. These cells express some or all of the receptors that permit viral binding, internalization, and infection, including CD4, CCR5, CXCR4 and DC-SIGN. Blocking the interaction between HIV-1 and infectivity receptors on target cells is an effective mechanism to inhibit HIV-1 infection. ${ }^{4}$ This has been successfully demonstrated using antibodies, ${ }^{5}$ soluble proteins, ${ }^{6,7}$ and chemokines..$^{8-10}$ Some of these inhibitors are currently being tested pre-clinically as microbicidal agents to prevent HIV-1 sexual transmission. ${ }^{11-15}$ However, the delivery of compounds that act on the cell surface are often less effective in mucosal tissues due to local conditions that degrade soluble proteins, and to physical barriers that prevent such molecules from penetrating the mucus covering and reaching cells in the lamina propria. Thus, the challenge exists to develop effective and non-toxic microbicidal compounds to interfere with the interaction between HIV-1 and its target cells in mucosal tissues.

An alternative approach to inhibit the interaction between HIV-1 and its target cell in the female reproductive tract is to reduce the cellular expression of receptors and co-receptors important in viral binding. Support for this approach comes from the observation that individuals who are heterozygous for the delta32 CCR5 mutation are significantly less likely to become infected with HIV-1 after sexual exposure than are individuals who express the wild-type form of the receptor. ${ }^{16,17}$ This indicates that even a partial reduction in receptor availability can significantly impact a person's susceptibility to acquiring sexually-transmitted HIV-1 infection. Reducing expression of cellular receptors in tissues can be achieved by the use of RNA interference (RNAi). RNAi uses small segments of complementary RNA called short-interfering RNA (siRNA) that are 20-25 nucleotides in length, to bind specific mRNA sequences within a cell. These complexes are then targeted for degradation by the formation of an RNA-induced silencing complex. siRNA sequences are highly specific and found to be efficacious in reducing expression of targeted genes. The use of RNAi is an attractive mechanism for reducing targeted gene expression in a therapeutic setting, is already in use clinically to treat including macular degeneration, ${ }^{18}$ and is currently under development for metabolic diseases, ${ }^{19}$ cancer, ${ }^{20}$ and viral dis-
Correspondence: Alexandra L. Howell, V.A. Medical Center, 215 North Main St. White River Jct., VT, USA 05009.

Tel. +1.802.295-9363 - ext: 5612 -

Fax: +1.802.296-5173.

E-mail: alexandra.howell@dartmouth.EDU

Key words: heterosexual transmission, women, virus infection, HIV-1, nanoparticles.

Acknowledgements: this work was supported by a Merit Review from the Department of Veterans Affairs (ALH) and a grant from the Veterans Education and Research Association of Northern New England (SKE).

Contributions: SKE, conceived, designed, performed molecular studies, tissue explant culture studies, transfection studies, HIV infection studies; NOI, performed the in vivo studies, analyzed and interpreted the data; JEC, designed transfection studies on mononuclear cell cultures, performed and analyzed the IFN- $\alpha$ studies, assisted SKE in molecular tissue explants analysis; SC, performed all analyses on mononuclear cell secretion of IFN- $\alpha$ by ELISA analysis; ALH, Principal Investigator in whose laboratory these studies were performed, responsible for the overall study design and data interpretation. Each author was responsible for designing, performing and interpreting the studies in their area as described above, and assisted in the writing and editing of relevant methods, results and discussion sections of the manuscript.

Conflict of interest: the authors report no conflicts of interest.

Received for publication: 1 March 2011

Revision received: 3 August 2011.

Accepted for publication: 3 August 2011.

This work is licensed under a Creative Commons Attribution NonCommercial 3.0 License (CC BYNC 3.0).

(C) Copyright S.K. Eszterhas et al., 2011

Licensee PAGEPress, Italy

Infectious Disease Reports 2011; 3:e11

doi:10.4081/idr.2011.e11

eases including HIV-1. ${ }^{21}$

Given the potential to specifically silence any gene of interest, siRNA offers several advantages over conventional drugs. To transfect the siRNA into cells, siRNA sequences are encapsulated into nanoparticles using various molecules that are readily endocytosed by phagocytic cells. ${ }^{22}$ Earlier studies using RNAi to inhibit HIV-1 infection employed siRNA sequences that silenced expression of viral sequences including Rev, Nef and Tat. ${ }^{23-25}$ Silencing cellular genes important for HIV-1 infection has also been explored. Martinez, et al. used siRNA to CXCR4 and CCR5 to reduce cell surface protein expression of these HIV-1 
co-receptors in tissue culture cells. The inhibitory effect of gene silencing was detected $48 \mathrm{~h}$ after transfection and reduced receptor expression in over one-half of receptor-positive cells. ${ }^{26}$ The suppression of receptor expression effectively blocked the acute infection of CXCR4 ${ }^{+} \mathrm{U} 87$ cells by X4 (HIV-1 $\mathrm{NL}_{\mathrm{NL}-3}$ ) and CCR5+ U87 cells by R5 (HIV-1 BaL ) HIV-1 strains. Arrighi, et al. used short hairpin RNA (shRNA)-expressing lentiviral vectors to inhibit the attachment of HIV-1 gp120 to DC-SIGN, as well as to inhibit the transfer of HIV-1 to target cells in trans. ${ }^{27}$

RNAi-mediated CCR5 silencing was achieved in vivo in a humanized murine model by incorporating integrin-targeting sequences into liposome particles that encapsulated CCR5-specific siRNA. ${ }^{28}$ These nanoparticles were targeted specifically to leukocytes by binding to the integrin-binding receptor, LFA1 , present on these cells. Animals who received an intravenous inoculation of these nanoparticles prior to intraperitoneal challenge with HIV-1 demonstrated a resistance to infection as determined by reductions in plasma viral load and maintenance of CD4 counts compared to untreated animals. The potential to target siRNA specifically to T cells in vivo was reported by Kumar, et al. ${ }^{29}$ siRNA to CCR5 was encapsulated in nanoparticles and these nanoparticles were complexed to an antibody with specificity for the pan T cell marker, CD7, to target siRNA to T cells in vivo. This modified siRNA was infused into human $\mathrm{CD} 34^{+}$progenitor cellreconstituted animals together with antibody to CCR5, prior to the intravenous infusion of HIV-1. The combination of an antibody to CCR5 to block HIV-1 binding to CCR5 together with siRNA to down-regulate CCR5 expression suppressed HIV-1 infection and prevented CD $4{ }^{+} \mathrm{T}$ cell loss in this animal model. ${ }^{29}$ To assess whether this technology could be useful clinically in patients already infected with HIV-1, mice were instead reconstituted with peripheral blood cells from an HIV-infected patient who was on anti-viral therapy and who had an undetectable viral load. The infusion of CCR5silencing siRNA nanoparticles targeted to $\mathrm{T}$ cells with anti-CD7 antibody maintained levels of $\mathrm{CD} 4{ }^{+}$cells in the bloodstream and kept viral load levels in the undetectable range, compared to mice that received an irrelevant siRNA. Nanoparticle delivery has also been explored as an approach to deliver microbicidal compounds and other anti-viral therapeutics to mucosal tissues of the human female reproductive tract. ${ }^{30}$ The encapsulation of bioactive compounds within nanoparticles not only protects these agents from enzymatic destruction by the acidic $\mathrm{pH}$ and enzymes of the vagina, but allows rapid and targeted uptake by phagocytic cells in the lamina propria. ${ }^{31}$ Thus, the development of nanoparticle transfection for the delivery of siRNA, bioactive compounds, and vaccine antigens to the mucosal tissues of the female reproductive tract could significantly advance therapeutic approaches to combat HIV-1 infection in these tissues. As HIV-1 vaccine trials have failed to produce a protective vaccine, the development of microbicidal compounds that can protect women from sexuallytransmitted infection have received considerably more attention in recent years. ${ }^{12}$

In this study, we sought to determine the efficacy of RNAi to inhibit HIV-1 infection in human female reproductive tract tissue explants. We used a novel primary tissue explant model ${ }^{32}$ in which explants from the human female reproductive tract were transfected with nanoparticles to deliver CD4 and CCR5-specific siRNA, and then challenged with HIV-1. The small size of these nanoparticles promoted endocytosis by phagocytic cells in the explants, thus transfecting target cells with the siRNA. Our findings demonstrate significant inhibition of HIV-1 infection in explants prepared from the endometrium, endocervix and ectocervix obtained from HIV-1 seronegative women undergoing hysterectomy for benign conditions. To determine the ability of siRNA nanoparticles to penetrate mucosal tissues in vivo, we assessed their efficacy to silence murine $\mathrm{Cd} 4$ after instillation into the reproductive tract of female mice. Significant silencing of murine $\mathrm{Cd} 4$ expression was detected in uterine tissues on day 3 after a single application of siRNA nanoparticles. In addition, we demonstrate that siRNA transfection induced the expression of IFN- $\alpha$, a potent antiviral cytokine that may contribute to the anti-HIV effect. These findings suggest that RNAi to silence cellular genes important for HIV-1 infection in mucosal tissues can be

developed as a potential therapeutic approach to inhibit HIV-1 transmission resulting from heterosexual exposure.

\section{Materials and Methods}

\section{Female reproductive tract tissues}

Female reproductive tract tissues were obtained from HIV-seronegative women undergoing hysterectomy at Dartmouth-Hitchcock Medical Center for benign conditions including fibroids, prolapse and dysmenorrhea. Informed consent was obtained prior to surgery. The age, diagnosis, menstrual cycle stage and hormonal therapy at the time of hysterectomy of each subject are shown in Table 1. Tissues were dissected free of underlying musculature within 4 hours of surgery, and the mucosal layers comprising both the epithelium and lamina propria were cut into $3 \mathrm{~mm}^{3} \mathrm{sec}$ tions. Two sections from each tissue site were placed into wells of a 48-well plate and cultured in $0.5 \mathrm{~mL}$ of Leibovitz's medium (L-15, Invitrogen, Carlsbad, CA, USA) containing 10\% heat-inactivated FBS, $2 \mathrm{mM}$ glutamine, 50 units/mL penicillin and $50 \mu \mathrm{g} / \mathrm{mL}$ streptomycin (Invitrogen), at $37^{\circ} \mathrm{C}, 5 \% \mathrm{CO}_{2}$ in a humidified atmosphere.

\section{siRNA transfection of tissue explants}

siRNA specific for human CD4 (s225098), CCR5 (s3211), or an irrelevant sequence (4390843), were obtained from Applied Biosystems/Ambion (Carlsbad, CA, USA). The encapsulating/transfection reagent INTERFER-

Table 1. Clinical summary of hysterectomy donors.

\begin{tabular}{|c|c|c|c|}
\hline Subject ID\# & Age & $\begin{array}{c}\text { Diagnosis } \\
\text { resulting } \\
\text { in hysterectomy }\end{array}$ & $\begin{array}{c}\text { Menstrual cycle } \\
\text { stage/Hormone } \\
\text { therapy }\end{array}$ \\
\hline $\begin{array}{l}\text { For CCR5 and CD4 transcription } \\
5041 \\
5063 \\
5082 \\
5165 \\
5177 \\
5210 \\
5995\end{array}$ & $\begin{array}{l}39 \\
55 \\
40 \\
52 \\
55 \\
62 \\
48\end{array}$ & $\begin{array}{l}\text { Fibroids } \\
\text { Uterine bleeding } \\
\text { Prolapse } \\
\text { Menorraghia } \\
\text { Fibroids } \\
\text { Prolapse } \\
\text { Pelvic Pain }\end{array}$ & $\begin{array}{c}\text { Secretory/none } \\
\text { Unknown/none } \\
\text { Secretory/none } \\
\text { Unknown/none } \\
\text { Atrophic/none } \\
\text { Atrophic/estrace cream* } \\
\text { Unknown/none }\end{array}$ \\
\hline $\begin{array}{l}\text { For HIV-1 Reverse transcription } \\
5207 \\
5210 \\
5233 \\
5254\end{array}$ & $\begin{array}{l}49 \\
62 \\
44 \\
52\end{array}$ & $\begin{array}{l}\text { Prolapse } \\
\text { Prolapse } \\
\text { Fibroids } \\
\text { Fibroids }\end{array}$ & $\begin{array}{c}\text { Secretory/none } \\
\text { Atrophic/estrace cream } \\
\text { Inactive/Lupron depot* } \\
\text { Unknown/none }\end{array}$ \\
\hline $\begin{array}{l}\text { For IFN- transcription } \\
5805 \\
5823 \\
5879\end{array}$ & $\begin{array}{l}67 \\
47 \\
59\end{array}$ & $\begin{array}{l}\text { Prolapse } \\
\text { Fibroids } \\
\text { Prolapse }\end{array}$ & $\begin{array}{l}\text { Atrophic/none } \\
\text { Unknown/alesse-28* } \\
\text { Unknown/none }\end{array}$ \\
\hline
\end{tabular}

*Estrace cream is a synthetic form of topically applied estrogen commonly used to treat vaginal atrophy; Lupron depot is a gonadotropinreleasing hormone sometimes used before hysterectomy in women with menorrhagia and anemia to prevent further bleeding prior to surgery; Alesse-28 is a synthetic progesterone and estradiol combination used as a contraceptive. 
in was obtained from Genesee Scientific (San Diego, CA, USA). INTERFERin carries a net positive charge, and when combined with the negatively charged siRNA molecules under vigorous vortexing forms nanoparticles that encapsulate the siRNA. Nanoparticles prepared in this manner and then stained with uranyl acetate and visualized by transmission electron microscopy are between 45 and $60 \mathrm{~nm}$ in diameter ${ }^{33}$ Nanoparticles were prepared by vortexing a solution containing 6 pmoles of siRNA, $2.4 \mu \mathrm{L}$ of INTERFERin, and $100 \mu \mathrm{L}$ of serum free media, followed by 15 min incubation at room temperature. This nanoparticle suspension was added to each well of tissue explants or peripheral blood mononuclear cells (PBMC) to give a final concentration of $20 \mathrm{nM}$ siRNA. Untreated tissue explants received only $100 \mu \mathrm{L}$ of the serum-free media per well. Twenty-four hours later, wells containing tissue explants were supplemented with $0.5 \mathrm{~mL}$ of fresh media (Figure 1). The following day, tissue explants were washed extensively and suspended in fresh media.

\section{HIV-1 infection of tissue explants}

Some of the issue explants were exposed to cell-free HIV- $1_{\mathrm{Ba}-\mathrm{L}}$ at a final virus concentration of $200 \mathrm{TCID}_{50} /$ well $48 \mathrm{~h}$ after siRNA transfection (Figure 1). This concentration of virus was previously determined to be optimal for infecting tissue explants (data not shown). Viral stocks were treated with 10 units/mL of RNase-free DNase-I prior to use to destroy any contaminating genomic DNA. After overnight incubation with virus at $37^{\circ} \mathrm{C}$, the female reproductive tract tissue sections were washed three times to eliminate non-internalized virus, and replenished in L15 medium.

\section{Nucleic acid isolation}

RNA was isolated from uninfected human tissue explants treated with siRNA nanoparticles to assess the magnitude of CCR5 and CD4 gene silencing. The replicate explants, plus any cells that migrated out of the tissue explant into the surrounding media (migratory cells), were collectively harvested after siRNA transfection and stored in RNAlater (Ambion, Austin, TX, USA) at -80o C until processing. Endocervix and endometrial tissues were harvested on days 3 and 6 post-silencing, whereas ectocervix tissue sections were harvested and assessed more frequently at days 1 , 2, 3, 4, 6 and 8 post-silencing. Tissues were thoroughly homogenized in RLTplus lysis buffer (Qiagen, Valencia, CA, USA) with a scintered glass homogenizer (Kontes, Vineland, NJ, USA). The lysate was subjected to RNA isolation using an RNeasy-Plus kit (Qiagen).

DNA was isolated from tissue explants and from migratory cells in culture on day 5 after infection (corresponding to day 8 post-siRNA transfection) using lysis buffer from Gentra PureGene Tissue Kit (catalog 158667, Qiagen) and columns from QIAamp DNA extraction kit (catalog 51306, Qiagen). DNA was analyzed by real-time PCR for HIV-1 reverse transcripts.

\section{CD4 and CCR5 transcription}

CD4 and CCR5 transcription was quantified in triplicate using real-time PCR with primers complementary to human CD4 and CCR5 genes. cDNA was produced from $2 \mu \mathrm{g}$ of cellular RNA using Superscript III reverse transcriptase (Invitrogen) and random hexamers (Invitrogen). To detect CD4 transcripts, the sense primer (5'-GGGGATACAGTGGAACTGACC3 ') and the anti-sense primer (5'-TCCCAAAGGCTTCTTCTTGAG-3') were used. For CCR5 transcripts, the sense primer (5'-GGCCAGAAGAGCTGAGACATCC-3') and the anti-sense primer (5'-CGGGCTGCGATTTGCTT CACAT-3') were used. In Figure 2, CD4 and CCR5 transcription levels were normalized to glyceraldehyde 3-phosphate dehydrogenase (GAPDH) transcripts (sense primer 5'-GGACCTGACCTGCCGTCTA-3', anti-sense primer 5'-TGCTGTAGCCAAATTCGTTG-3') and relative expression was determined using $2{ }^{-}{ }^{\Delta} \mathrm{C}_{\mathrm{T}}$ analysis. In Figure 3, CD4 and CCR5 transcription levels were normalized to GAPDH transcripts, and presented as arbitrary units using $2-{ }^{-} \mathrm{C}_{\mathrm{T}}$ analysis.

\section{HIV-1 reverse transcription}

HIV-1 reverse transcription was detected with primers complementary to both the reverse transcription product and the integrated virus. Genomic DNA (50-200 ng) isolated from HIV-1 exposed tissue sections was subjected to real-time PCR using the sense primer (5'-GGAACCCACTGCTTAAGCCTCAA-3') and

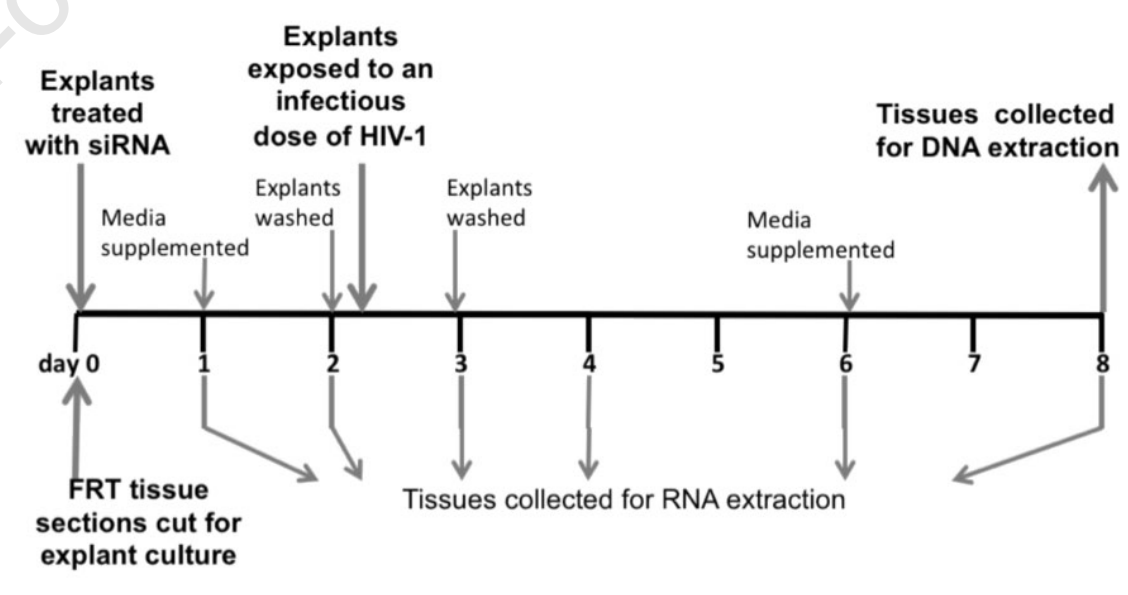

Figure 1. Timeline of the experimental protocols using human female reproductive tract explants. Samples of female reproductive tract tissues were received, dissected, and placed into culture on day 0 , the same day they were transfected with nanoparticles containing siRNA. On day 2 , the explants were thoroughly washed to remove residual siRNA nanoparticles, and some sections were exposed to cell-free HIV-1BaL. On day 3, the HIVexposed explants were again thoroughly washed to remove any unincorporated HIV-1. CCR5 and CD4 mRNA levels were measured on uninfected ectocervical explants on days $1,2,3,4,6$, and 8 , and only on days 3 and 6 in explants from endometrium and endocervix. HIV-1 DNA was measured on the HIV-1 exposed explant cultures on day 8. the anti-sense primer (5'-TGTTCGGGCGCCACTGCTAGAGA-3'). ${ }^{34}$ HIV-1 reverse transcript levels were normalized to genomic human $\beta$ actin amplified with the sense primer (5'3 ') and relative starting quantity was determined using $2-{ }^{-{ }_{\Delta}} \mathrm{C}_{\mathrm{T}}$ analysis.

\section{Murine siRNA studies}

All animal components of the research protocols (ACORP) were approved and supervised by our Institutional Animal Care and Use Committee (IACUC). Six to 8 week old female $\mathrm{Balb} / \mathrm{C}$ mice were obtained from Harlan Laboratories (South Easton, MA, USA). anoparticles for in vivo use were prepared by ratio of 8 . Forty $\mu \mathrm{L}$ of the suspension containing $40 \mu \mathrm{M}$ of $\mathrm{Cd} 4$ specific siRNA (s63657, Applied Biosystems/Ambion) or an irrelevant siRNA (ss20212, Applied Biosystems/Ambion) was instilled separately into each uterine horn inserting it atraumatically into the vaginal canal and past the cervical os, directing the solution first into one uterine horn, then reinserting a second application of an identical volume into the other uterine horn. Mice were anesthetized with inhalation isofluorane immediately prior to and during the instillation, and were kept anesthetized and in a head vent nal canal. Two to four mice from each experimental group (Cd4 or irrelevant siRNA) were euthanized by $\mathrm{CO}_{2}$ inhalation on days $1,2,3$, and 5 post-siRNA instillation, and reproductive

\section{.} CACTCTTCCAGCCTTCCTTCC-3') and the antiin vivo Jet PEI (GeneSee Scientific) at an N/P 
organs (vagina plus cervix, uterine horns) were removed and stored in RNAlater (Qiagen, Valencia, CA, USA) at $-80^{\circ} \mathrm{C}$. The tissues were then thawed and homogenized, and RNA isolated as described ${ }^{35}$ Real time PCR was used to quantitate $\mathrm{Cd} 4$ using murine specific $\mathrm{Cd} 4$ primers (5'-TGCAAACACAAAAAGGGTAAA-3' and 5'-TACGACCAGAGGCATACAGGGACAG-3'), and normalized to murine $\beta$-actin (sense primer 5'-ACCAACTGGGACGACATGGAGAAGA3 ' and anti-sense primer 5' - TACGACCAGGAGGCATACAGGGACAG-3').

\section{PBMC isolation}

Peripheral blood was obtained after informed consent from normal donors, and the mononuclear cell fraction isolated by FicollPaque (Amersham, Piscataway, NJ, USA) as described. ${ }^{35}$ PBMC were suspended to $2 \times 10^{6}$ cells/mL in RPMI-1640 supplemented with $10 \%$ heat-inactivated FBS, $2 \mathrm{mM}$ glutamine, 50 units/mL penicillin and $50 \mu \mathrm{g} / \mathrm{mL}$ streptomycin (GIBC0). Two million cells were added to wells of a six-well plate prior to transfection with siRNA nanoparticles.

\section{IFN- $\alpha$ secretion from siRNA-treat- ed PBMC and tissue explants}

Supernatants were collected from PBMC exposed to siRNA nanoparticles, or from PBMC left untreated, immediately prior to siRNA transfection, and again at 4, 24, 48 and $96 \mathrm{~h}$ post-transfection. Levels of IFN- $\alpha$ in the supernatant were measured by ELISA ( $R$ and $D$ Systems, Minneapolis, MN, catalog \#41105-1, IFN- $\alpha$ Multi-Subtype ELISA Kit).

As the levels of secreted IFN- $\alpha$ from tissue explants were below the level of detection of the IFN- $\alpha$ ELISA kit, we quantified expression of IFN- $\alpha$ from ECX tissue sections by realtime PCR. ECX tissues were chosen for study because this tissue type has the highest concentration of leukocytes compared to other sites within the female reproductive tract. ${ }^{32}$ RNA isolated from siRNA-treated ECX tissue explants were subjected to stringent removal of contaminating DNA prior to amplification. IFN- $\alpha$ transcripts were amplified with the sense primer (5'- GCA CCG AAC TCT ACC AGC AGC-3') and anti-sense primer (5'- TCT GAC AAC CTC CCA GGC ACA-3'). These primers amplify a product that is 179 base pairs in size. $^{36}$

\section{Statistical analysis}

Analysis of datasets comparing two groups was performed by students' T-test, and those comparing multiple groups were performed by ANOVA, and were considered statistically significant at $\mathrm{P}<0.05$.

\section{Results}

siRNA silences CD4 and CCR5 expression in female reproductive tract tissue explants after a single siRNA exposure

CD4 and CCR5 expression was measured in endometrial (EM) and endocervical (CX) explants on days 3 and 6 post-siRNA transfection, and on ectocervical (ECX) explants on days at $3,4,6$ and 8 post-siRNA transfection. For each subject, expression levels from CD4 and CCR5-specific siRNA-treated explants were normalized to sections treated with irrelevant siRNA to control for the effects of nanoparticle transfection on cells. We detected significant reductions in CD4 transcript levels in EM and ECX, but not in CX, in response to the combination of CD4 and CCR5 specific siRNA (Figure 2A). For example, CD4 expression was suppressed by approximately $45 \%$ in explants from the EM and ECX on day 6, and even more strongly suppressed (approximately $60 \%$ ) in ECX on day 4 (Figure 2A). In contrast, CCR5 expression showed a different pattern. CCR5 was suppressed by specific siRNA on day

A.

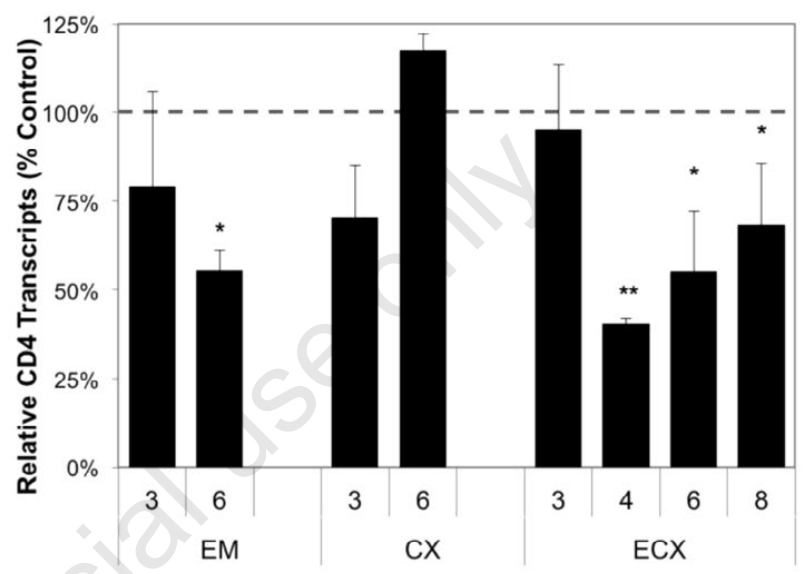

Days Post siRNA Treatment of Explants

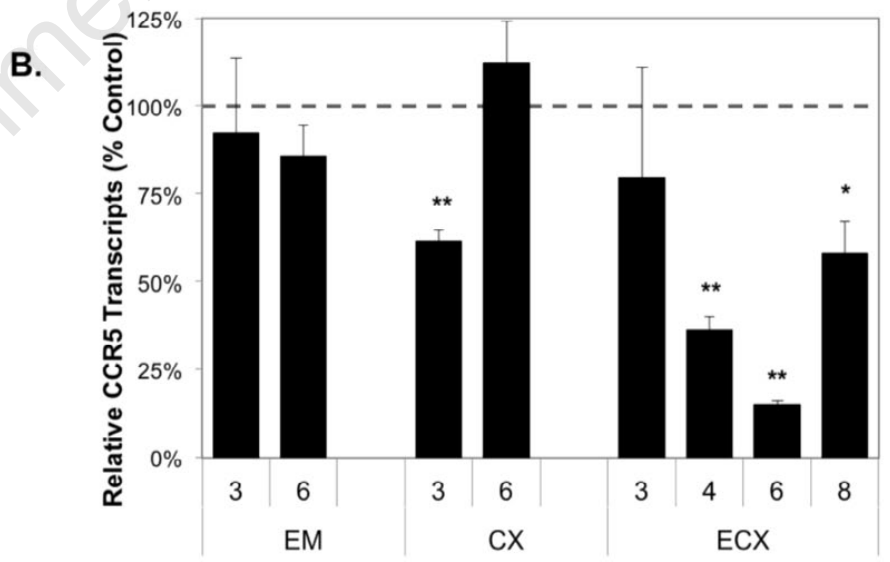

Days Post siRNA Treatment of Explants

Figure 2. siRNA suppresses both CD4 and CCR5 expression in human female reproductive tract explants. Female reproductive tract tissue explants from the endometrium (EM) endocervix $(\mathrm{CX})$ and ectocervix $(\mathrm{ECX})$ were treated with nanoparticles containing either an irrelevant siRNA $(20 \mathrm{nM})$ or a combination of CCR5- and CD4-specific siRNA (10 $\mathrm{nM}$ each, black bars). Shown are CD4 (Panel A) and CCR5 (Panel B) expression on days 3 and 6 for EM and CX, and days 3, 4, 6 and 8 for ECX. The data represent the average of the mean relative expression of 6 subjects for CD4 and 4 subjects for CCR5. The expression from each subject was assayed in triplicate, normalized to GAPDH expression, and expressed relative to values for explants treated with irrelevant siRNA from that patient (irrelevant siRNA control $=\mathbf{1 0 0} \%$ ). The bars represent the average of the relative mean expression levels from 4 or 6 subjects and the error bars are the standard deviation of the means. A single asterisk $\left({ }^{*}\right)$ denotes statistically significant differences at $\mathbf{P}<0.05$ and double asterisks $\left.{ }^{* *}\right)$ denotes statistically significant differences at $\mathrm{P}<0.005$ between explants treated with irrelevant and receptor-specific siRNAs. Bars with no asterisk did not reach statistical significance at the $95 \%$ confidence level. 
3 in the CX but this suppression was not sustained out to day 6 . In the ECX, CCR5 was not suppressed until day 4 but its suppression was sustained throughout the 8 days of culture (Figure 2B) reaching a maximum suppression of approximately $85 \%$ on day 6 compared to the irrelevant siRNA control. EM was refractory to suppression of CCR5 on both day 3 and 6 (Figure 2B).

\section{Time course of siRNA silencing of CD4 and CCR5 in ectocervical explants}

We performed a detailed time course of CD4 and CCR5 transcript levels in ectocervical explants from a single subject to more closely assess comparisons among untreated, irrelevant siRNA and specific siRNA treatment on transcript levels. CD4 transcript levels in explants left untreated showed a drop over the first 3 days of culture and then a leveling off throughout the remainder of the culture period (Figure 3A). Explants treated with an irrelevant siRNA nanoparticle showed a similar initial decrease in CD4 transcript levels and fluctuations in levels from days 4 through 8 of culture. In contrast, explants treated with nanoparticles containing the combination of CD4- and CCR5siRNA showed a sustained suppression of CD4 transcript levels throughout the 8-day culture period (Figure 3A). Analyses of CCR5 transcript levels indicate that in the untreated explants, there is a similar initial decrease in transcript levels in the first 3 days of culture, with a leveling off throughout the remainder of the culture period (Figure 3B). Explants treated with an irrelevant siRNA showed a marked and reproducible increase in CCR5 transcript levels on day 4 post-transfection. This increase was followed by a down-regulation on days 6 and 8 . In contrast, explants treated with the combination of CD4- and CCR5-siRNAs demonstrated a reduced level of CCR5 transcripts starting on day 3 when compared to both the untreated and irrelevant siRNA-treated explants (Figure 3B). The receptor-specific siRNAs ablated any nonspecific stimulatory effects on both CD4 and CCR5 expression observed with the irrelevant siRNA containing nanoparticles.

\section{HIV-1 infection is reduced in female reproductive tract explants silenced for CD4 and CCR5}

Female reproductive tract explants left untreated, or transfected with nanoparticles containing either an irrelevant siRNA or the combination of siRNAs to CD4 and CCR5, were evaluated for HIV-1 reverse transcripts on day 8 of culture (day 5 post-infection). HIV-1 DNA levels were normalized to those observed in the irrelevant siRNA nanoparticle-treated explants. We observed a significant inhibition of HIV-1 reverse transcripts in all three tissue types (EM, CX and ECX) following transfection with specific siRNAs, compared to those explants treated with the irrelevant siRNA (Figure 4). CX explants treated with the combination of CD4- and CCR5-specific siRNAs showed only a modest inhibition of HIV-1 infection (approximately 40\%), whereas ECX explants had a very robust inhibition (approximately 80\%) following CD4- and CCR5-siRNA treatment. For both the CX and ECX, HIV-1 infection in explants left untreated was approximately equivalent to the irrelevant siRNA treated explants. In contrast, HIV-1 infection in the untreated EM explants showed lower levels of HIV-1 reverse transcripts compared to those EM explants treated with the irrelevant siRNA nanoparticles. However, even in the EM, HIV-1 infection was significantly reduced in explants treated with nanoparticles containing the combination of CD4- and CCR5siRNAs when compared to those treated with the irrelevant siRNA. In contrast, in the CX and ECX, treatment with the irrelevant siRNA nanoparticles did not increase HIV-1 transcript levels compared to the untreated explants. Thus, we observed a significant decrease in HIV-1 transcripts in all explants treated with nanoparticles containing the combination of
A.

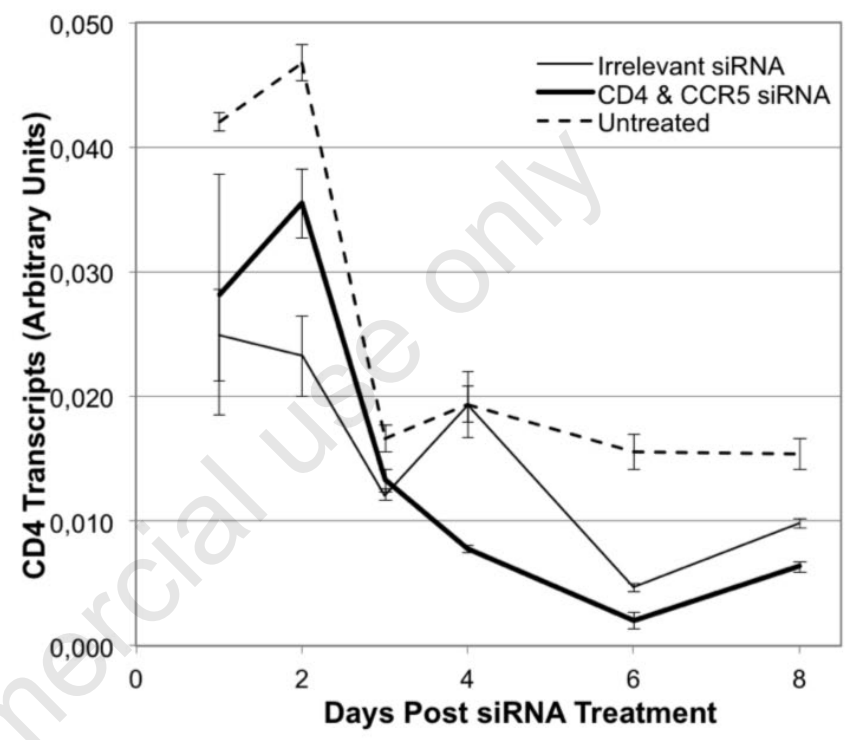

B.

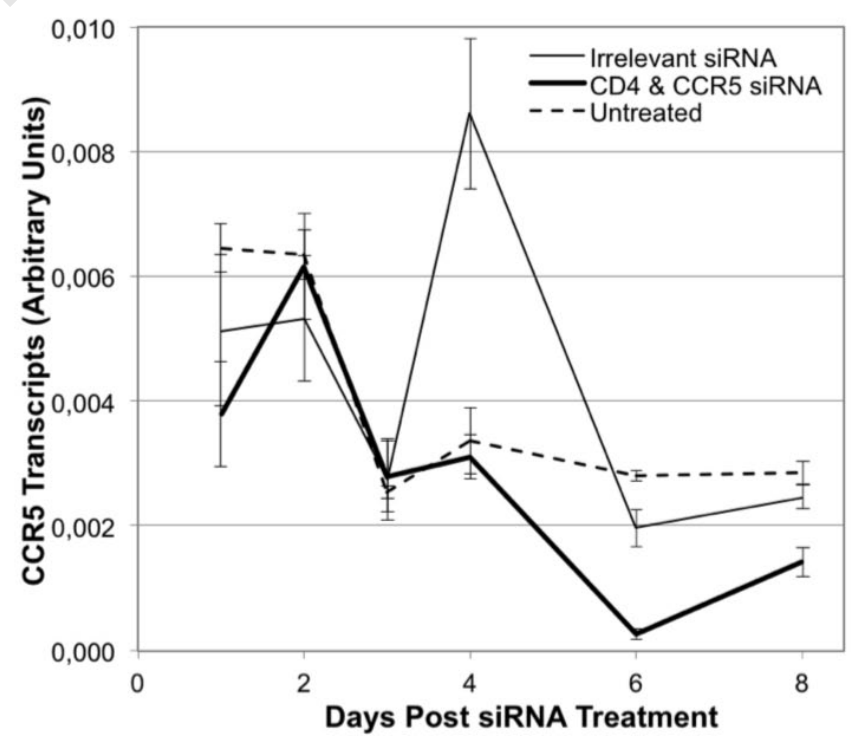

Figure 3. Timeline of siRNA effects on CD4 and CCR5 expression in human female reproductive tract ectocervical explants. Ectocervical explants were treated with siRNA nanoparticles containing irrelevant siRNA (20 nM, thin line), the combination of both CD4- and CCR5-specific siRNAs (10 nM each, heavy line), or left untreated (dashed line). On days 1, 2, 3, 4, 6 and 8 of culture, tissues were harvested and assessed for levels of CD4 transcripts (panel A, top) or CCR5 transcripts (panel B, bottom). The data is from a single representative subject and shows transcript levels in arbitrary units normalized to expression of GAPDH. Error bars show standard deviation of triplicate assays. 
receptor specific-siRNAs compared to the irrelevant siRNA (Figure 4).

\section{siRNA nanoparticles silence murine $\mathrm{Cd} 4$ in reproductive tract tissues in vivo}

To determine the efficacy of RNA interference using siRNA nanoparticles in an intact and functioning reproductive tract, nanoparticles containing murine Cd4-specific or irrelevant siRNA were instilled directly into both uterine horns of female mice. Uterine tissues analyzed on day 3 post-Cd4 siRNA nanoparticle instillation showed a significant reduction in murine $\mathrm{Cd} 4$ expression when compared to tissues from the irrelevant siRNA-treated control mice $(\mathrm{P}<0.05$, Figure $5 \mathrm{~A})$ although this silencing effect was not observed at day 5 . To determine whether the reduction in $\mathrm{Cd} 4$ transcript levels may have resulted from fewer numbers of murine leukocytes in the uterus of $\mathrm{Cd} 4$ siRNA-treated mice, we analyzed murine Cd45 transcript levels in these tissues. We observed no significant alterations in $\mathrm{Cd} 45$ transcript levels in uterine tissues obtained from mice treated with the Cd4-siRNA nanoparticles compared to those treated with the irrelevant siRNA control. Using real time PCR to quantify Cd4 and Cd45 transcript levels, no significant reductions were detected in the vaginal/cervical tissues that were downstream of the nanoparticle instillation site (data not shown). Real time PCR data were normalized to murine $\beta$-actin.

\section{PBMC and tissue explants treated with siRNA nanoparticles secrete IFN- $\alpha$}

To determine mechanisms other than gene silencing that could contribute to the antiviral effect of siRNA transfection of tissue explants, we measured levels of IFN- $\alpha$ released into the culture supernatant from PBMC from cells left untreated, or treated with nanoparticles containing either an irrelevant siRNA or the combination of CD4- and CCR5-specific siRNAs. IFN- $\alpha$ secretion was detected using ELISA as early as 24 hours post-transfection in cells treated with nanoparticles containing either an irrelevant siRNA or CD4- plus CCR5-specific siRNAs. IFN- $\alpha$ was detected in the culture supernatant throughout the $72 \mathrm{hr}$ culture period (Figure 6A). No IFN- $\alpha$ secretion was detected at any time-point from the untreated PBMC.

Secretion of IFN- $\alpha$ by tissue explants was too low to detect by ELISA, so real-time PCR was used to measure expression of IFN- $\alpha$ transcripts in ectocervical explants. As shown in Figure $6 \mathrm{~B}$, we observed significant levels of IFN- $\alpha$ transcripts in ECX tissues treated with nanoparticles containing either irrelevant siRNA or the combination of CD4- and CCR5siRNAs by day 2 after transfection, and this effect was sustained to day 4 (Figure 6B). In both the PBMC cultures as well as the tissue explants, induction of IFN- $\alpha$ protein (PBMC) or its transcript (explants) was greater in cultures treated with nanoparticles containing the combination of CD4- and CCR5-siRNA as compared to the irrelevant siRNA.

\section{Discussion}

The female reproductive tract tissue explant model has been invaluable for studies of mucosal immunity, HIV-1 infection, and microbicidal efficacy. Despite limitations to using human female reproductive tract explants in these types of studies, ${ }^{37}$ the availability of tissues from clinically-defined subjects free of gynecological malignancy has allowed rapid advances in our understanding of the mechanisms involved in the early events in HIV-1 infection at this site, and in ways to block or otherwise inhibit viral infection either with vaccines or microbicides. We previously demonstrated that tissue explants prepared from the female reproductive tract of seronegative women could be infected with HIV-1, and that the level of infection among female reproductive tract sites correlated with expression of the pan-leukocyte marker, CD45. ${ }^{32}$ In this study we exposed female reproductive tract explants to siRNA to silence CD4 and CCR5 for $48 \mathrm{~h}$ prior to the addition of HIV-1 to determine the efficacy of gene silencing to protect tissue explants from HIV-1 infection. We then measured the degree of gene silencing and of inhibition of HIV-1 reverse transcription on subsequent days of culture. Control tissue explants were treated with nanoparticles containing irrelevant siRNA to control for any effects on susceptibility to HIV-1 infection induced by nanoparticle uptake. Such effects have been reported and include cellular activation, effects on cellular viability, or bystander effects on the expression of non-targeted gene products. ${ }^{38-40}$ We found that silencing expression of CD4 and CCR5 in these explants using nanoparticles to deliver siRNA reduced expression of targeted gene products and inhibited HIV-1 reverse transcription. Thus, it is likely that reducing expression of receptors required by HIV-1 for infection can result in an inhibition of HIV-1 infection in mucosal tissues, including the female reproductive tract.

In human female reproductive tract

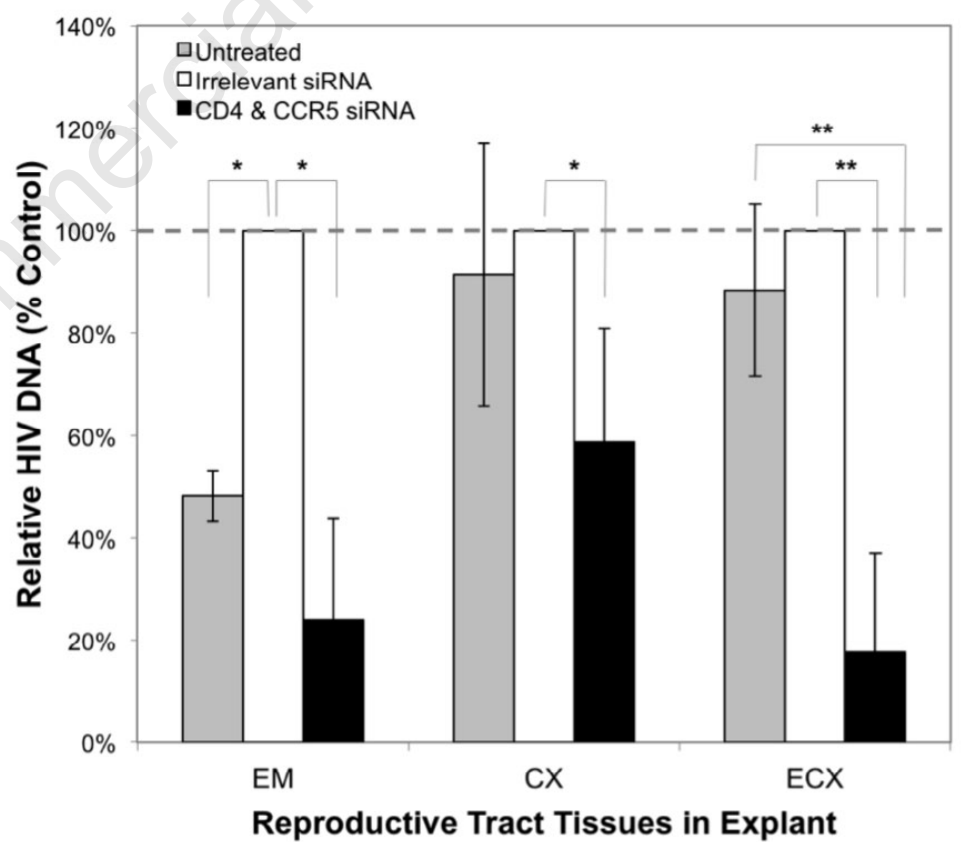

Figure 4. Nanoparticles containing CCR5 and CD4 specific siRNAs inhibit HIV-1 reverse transcription in female reproductive tract explants. Female reproductive tract tissue explants from the endometrium (EM), endocervix $(\mathrm{CX})$ and ectocervix $(\mathrm{ECX})$ were either left untreated (grey bars), or treated with nanoparticles containing irrelevant siRNA (20 nM, white bars), or the combination of both CCR5 and CD4-specific siRNA (10 nM each, black bars) for $48 \mathrm{hrs}$ prior to exposure to HIV-1BaL. Explants were assessed for HIV-1 reverse transcripts 5 days after virus exposure. Data are displayed by setting the levels of HIV-1 DNA from the irrelevant siRNA treated explants to $100 \%$. Data are a compilation from four subjects, each of which was assayed in triplicate. Error bars show the standard deviation of the means relative to the irrelevant siRNA control. The single asterisk $\left({ }^{*}\right)$ denotes statistically significant differences among bracketed bars at the $\mathbf{P}<0.05$ level, and the double asterisks $\left({ }^{* *}\right)$ denote significance at the $P<0.005$ level. Bars with no asterisks did not reach statistical significance at the $95 \%$ confidence level. 
explants, we observed that the kinetics and magnitude of gene silencing after a single application of siRNA nanoparticles varied among the three tissue sites (EM, CX and ECX). This finding is likely due in part to differences among tissue sites in the number and density of leukocytes present in the lamina propria. In our previous study, we determined that the ECX has the highest level of CD45 expression in the female reproductive tract, indicating an enrichment of leukocytes at this site. ${ }^{32}$ Moreover, we have found that the expression of CD4, CCR5, and CXCR4 varied in the ECX and in the EM on both the stromal leukocyte population as well as on epithelial cells, and the numbers and distribution of receptor-positive leukocytes also varied throughout the menstrual cycle, indicating hormonal regulation of receptor expression and leukocyte trafficking. ${ }^{41,42}$ Thus, the varied findings among tissue sites is likely due to multiple effects including the density of receptor-positive cells, or to donor variation in age, menstrual cycle stage and/or clinical conditions. In addition, the efficacy of siRNA delivery via nanoparticles may be impacted by the degree of mucus within the female reproductive tract, or to other physical barriers such as the thickness or the type of epithelium. Nanoparticles must penetrate through the mucus layer and reach target cells in the lamina propria in order to bind to cells and deliver the siRNA molecule. Moreover, columnar epithelium lines the tissues of the upper reproductive tract (CX and EM), and nanoparticles may penetrate through this single cell layer more easily than the stratified epithelial lining of the lower reproductive tract (ECX). Thus, the combined effects of target cell density, thickness of the mucus layer and type of epithelium are likely to contribute to the varied efficacy of RNA interference in the female reproductive tract. Although we did not measure the penetration or distribution of the nanoparticles into the tissue explants, we have determined using isolated populations from the peripheral blood that nanoparticles are selectively engulfed by monocytes and not by lymphocytes (data not shown). This may also be the case in tissues, and may contribute to the incomplete suppression of CD4 and CCR5 transcript levels, as these receptors are also expressed by lymphocytes in the lamina propria. Nonetheless, our findings indicate that cells within tissue explants do take up siRNA delivered within nanoparticles, and that the siRNA can effectively silence gene expression.

To determine if nanoparticles containing siRNA could penetrate mucosal tissues in vivo, we tested the efficacy of these particles to silence expression of $\mathrm{Cd} 4$ in the murine female reproductive tract. A single application of nanoparticles containing murine Cd4-specific siRNA instilled directly into both uterine horns significantly suppressed Cd4 expression only on day 3 post-transfection. We did not observe significant silencing prior to day 3 , and the effect was short lived in that the effect was largely gone by day 5 . This is likely due to the time required for penetration of nanoparticles in vivo, as well as to efficacy of nanoparticle uptake in receptor-positive cells. A more thorough assessment to identify the cell lineages that take up nanoparticles in vivo along with measurements of efficacy of gene silencing are currently underway and should contribute to dosage and dosing frequency for optimal gene silencing in vivo.

Interestingly, inhibition of HIV-1 reverse transcription by siRNA was most effective in the ECX, a female reproductive tract site that has a stratified epithelium and mucus secretion. This finding suggests that there may be a greater density of HIV-1 susceptible macrophages at this site, as macrophages readily endocytose nanoparticles. In all three tissue types, the use of receptor-specific siRNAs significantly inhibited HIV-1 transcription when compared to explants treated with an irrelevant siRNA nanoparticle. In the CX

A.
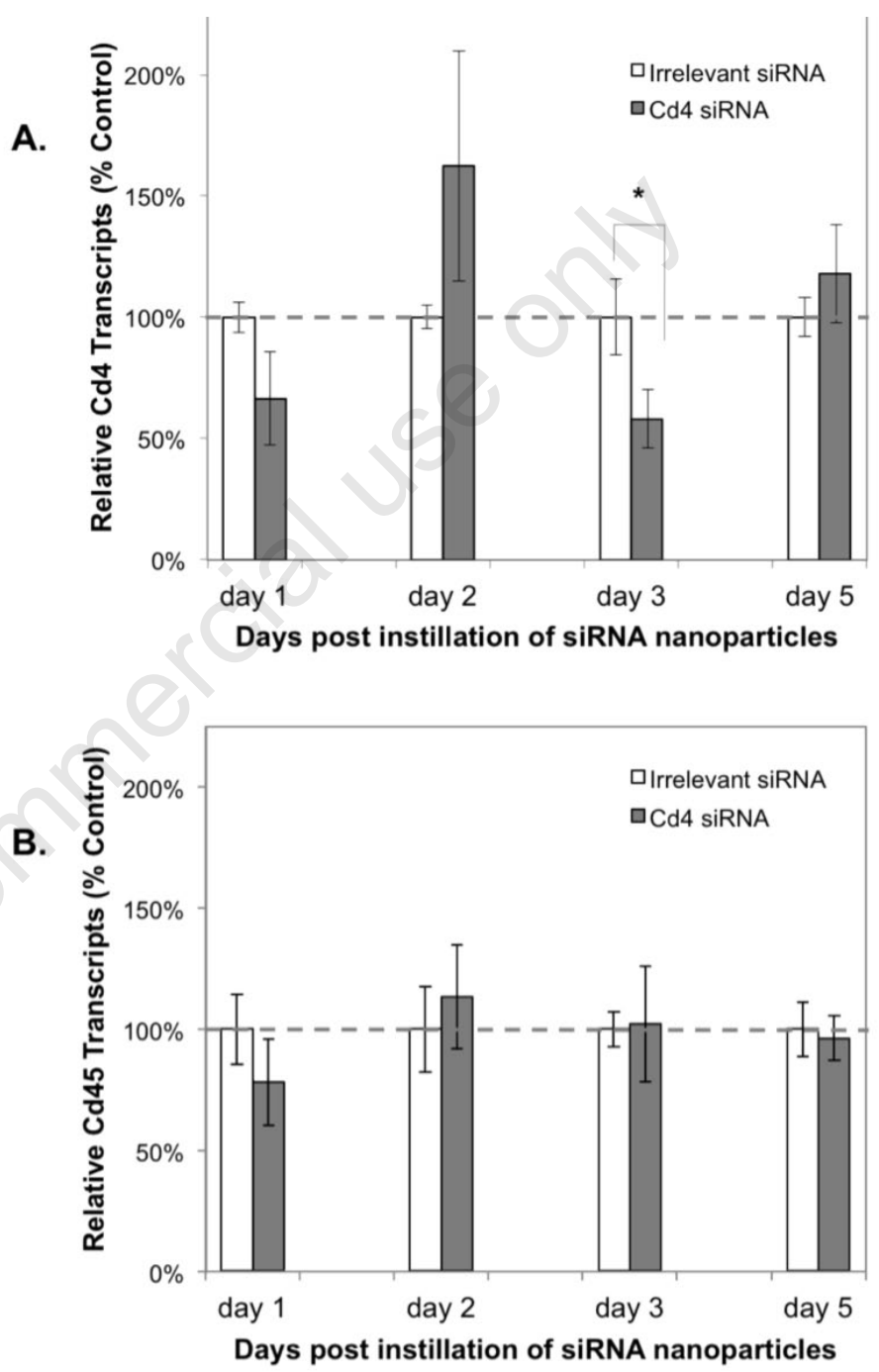

Figure 5. Nanoparticles containing siRNA to murine Cd4 reduce Cd4 expression but not Cd45 expression in murine uterine tissues. Nanoparticles containing siRNA to an irrelevant sequence or to murine $\mathrm{Cd} 4(10 \mathrm{\mu g} / \mathrm{mouse})$ were instilled directly into each uterine horn. On days 1, 2, 3 and 5, the mice were euthanized and uterine tissues assessed by real-time PCR for Cd4 expression (Panel A) or for Cd45 expression (Panel B) in triplicate and normalized to murine $\beta$-actin expression. Average relative expression of $\mathrm{Cd} 4$ or Cd45 was compared between groups of mice treated with the irrelevant siRNA control (white bars) or Cd4-specific siRNA (grey bars). Two mice are represented in each group for days one and two and four mice are represented in each group for days 3 and 5 . Error bars show the standard deviation of the means among the mice in each group. A statistically significant difference (as denoted by a single asterisk) between the irrelevant and Cd4-specific siRNA treated groups was observed on day $3(\mathrm{P}<0.05)$. Bars with no asterisk did not reach statistical significance at the $95 \%$ confidence level. 
and ECX, we observed no significant differences in the levels of HIV-1 reverse transcripts between explants that were left untreated and those treated with irrelevant siRNA nanoparticles. This was not the case in the EM, however, in which lower levels of HIV-1 reverse transcripts were measured in untreated explants compared to those treated with the irrelevant siRNA. One possible explanation for this finding is that exposure to siRNA containing nanoparticles enhances HIV-1 infectivity in the EM. Whether this results from differences in responsiveness to RNA molecules by innate immune cells at this normally sterile site, or to other off-target effects of nanoparticle transfection, is not known at this time. These findings do, however, show that the complete silencing of receptor expression is not necessary to inhibit HIV-1 infection. Alternatively, gene silencing of CD4 and CCR5 may inhibit the spread of HIV-1 infection in the tissues, rather than inhibit the initial infectivity event. Although we did not correlate the kinetics of CD4 and CCR5 transcript inhibition with the concomitant reduction in surface expression, it is likely that the uptake of nanoparticles containing siRNA serves to inhibit HIV-1 infection by mechanisms other than receptor suppression.

To determine whether other mechanisms may contribute to inhibiting HIV-1 infection in the female reproductive tract explants, we measured the release of IFN- $\alpha$, a potent antiviral cytokine, from PBMCs treated with siRNA nanoparticles. We chose to assess IFN- $\alpha$ production from nanoparticle-exposed cells for several reasons. Toll-like receptors (TLR)-7, 8 and 9 that belong to a class of innate immune receptors release anti-viral cytokines including IFN- $\alpha / \beta$ and $\lambda$ upon activation. ${ }^{43}$ These TLR are located in endosomes of phagocytic cells, and bind double- and single-stranded nucleic acids characteristic of viral and bacterial pathogens. As siRNA-encapsulated nanoparticles are taken up by cells in endocytic vesicles, it is likely that activation of these TLR would occur by binding to siRNA, leading to inflammatory cytokine production. ${ }^{33,44-47}$ Moreover, IFN- $\alpha$ has been associated with inhibiting HIV-1 replication in vitro, and with persistent HIV-1 infection in vivo. ${ }^{48,49}$ Thus, the potential for siRNA-nanoparticles to both silence gene expression and activate innate immune responses could serve to enhance protection from HIV-1 infection in the female reproductive tract. We found that IFN- $\alpha$ was released from PBMC within 24 hours of siRNA transfection, and that levels of IFN- $\alpha$ were detectable in culture supernatants throughout the 4 day culture. Moreover, irrelevant siRNA also induced the release of IFN- $\alpha$, although not to levels observed with receptor-specific siRNAs. No IFN- $\alpha$ was detected from untreated cells indicating a direct correlation between siRNA transfection and induction of this cytokine. Although we did not test whether the levels of secreted IFN- $\alpha$ were high enough to protect cells from HIV-1, it is possible that inflammatory responses to siRNA could contribute to the inhibition of HIV-1 infection in the female reproductive tract. Using real-time PCR, we found a similar induction of IFN- $\alpha$ transcription in ECX explants after siRNA treatment, but not until day 2 post-transfection. As with the PBMC cultures, both irrelevant and recep- tor-specific siRNA nanoparticles induced IFN$\alpha$ transcripts. Further studies are needed to determine the various mechanism(s) that are likely to contribute to HIV-1 inhibition of infection in female reproductive tract explants following siRNA transfection with nanoparticles.

In sum, our findings indicate that transfection of female reproductive tract tissue explants with siRNA-encapsulated nanoparticles results in a reduction of targeted gene expression and in inhibition of HIV-1 infection.
A.

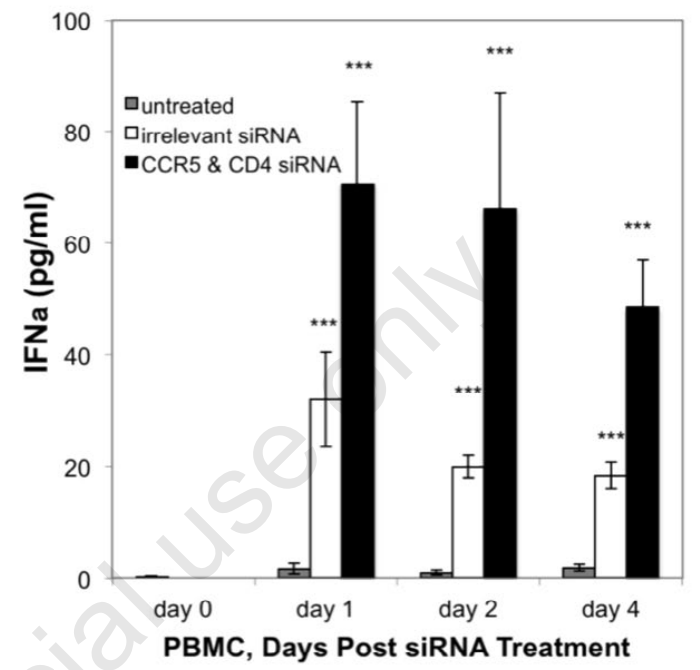

B.

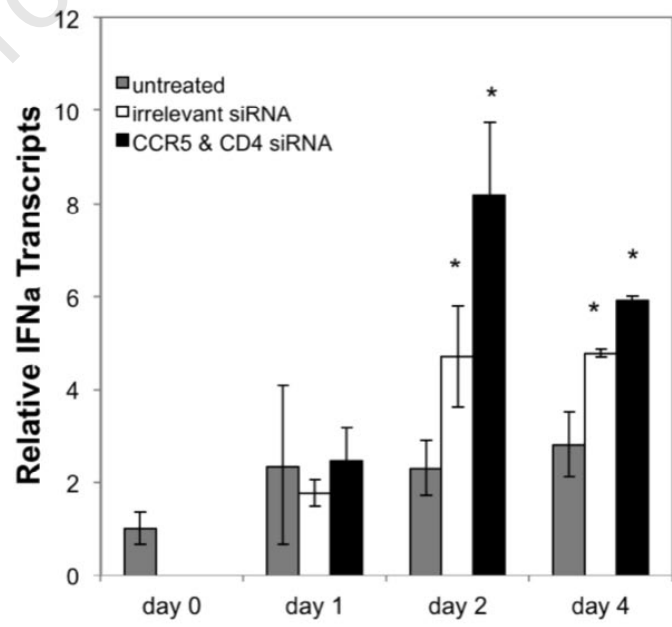

Ectocervix Explant, Days Post siRNA Treatment

Figure 6. siRNA transfection upregulates IFN- $\alpha$ expression. A) IFN- $\alpha$ secreted by PBMC left untreated (grey bars) or treated with nanoparticles containing irrelevant siRNA (20 $\mathrm{nM}$, white bars) or the combination of CCR5 \& CD4-specific siRNA (10 nM each, black bars) were quantified at time 0 , day 1 , day 2 , and day 4 post-transfection. Error bars show the standard deviation of the replicates. Asterisks $(* * *)$ denote statistically significant differences $(P<0.001)$ in IFN- $\alpha$ production between untreated and siRNA-treated PBMCs. B) siRNA stimulates IFN- $\alpha$ transcription in ectocervical explants. Total RNA was extracted from ECX explants on days 1, 2 and 4 either left untreated (grey bars), or after transfection with nanoparticles containing irrelevant siRNA ( $20 \mathrm{nM}$, white bars), or CCR5 plus CD4 specific siRNA (10 nM each, black bars). Levels are expressed as mean IFN- $\alpha$ transcripts relative to that observed at culture initiation (day 0). Error bars the show standard deviation of the means. Asterisks $\left(^{*}\right)$ denote statistically significant differences $(P<0.05)$ between explants treated with siRNA as compared to untreated explants. Bars with no asterisk did not reach statistical significance at the $95 \%$ confidence level. 
This is an important finding because the use of siRNA has been effective in treating ocular diseases $^{18}$ and nanoparticles have been determined to be safe for mucosal tissue delivery Moreover, the use of nanoparticles for microbicidal delivery to both the female reproductive tract and the rectal mucosa indicates the potential for this approach to target primary HIV-1 infection in mucosal tissues. ${ }^{12,50-52}$ The potential to knock down gene expression within the female reproductive tract and induce protection against a sexually transmitted disease could be developed not only for HIV-1, but also for other pathogens that utilize cell-specific receptors for infection. Thus, it is possible to engineer nanoparticles for the delivery of siRNA, either alone or in combination with anti-HIV drugs, to achieve protection against sexual HIV-1 infection. Such nanoparticles could be manufactured in either a gel, film or ring formulation, so that they are stable upon storage, easy to apply, and can be designed to be timed-released and thus coitally-independent. ${ }^{53,54}$ Nanoparticles can be designed to have optimal bio-distribution and residence time in mucosal tissues such that delivery of the bioactive components occurs throughout the lower reproductive tract (vaginal mucosa and cervix) at a continuous and stable rate. It is highly likely that nanoparticle-based microbicides can be designed with controlled architecture and surface chemistry that would retain efficacy in the mucosal tissues and allow delivery of active compounds without toxicity or damage to the epithelium. Such nanoparticle-based microbicides could provide HIV-1 protection for women until an effective HIV vaccine is developed.

\section{References}

1. Nunnari G, Otero M, Dornadula G, et al. Residual HIV-1 disease in seminal cells of HIV-1-infected men on suppressive HAART: latency without on-going cellular infections. AIDS 2002;16:39-45.

2. Kiessling A. Isolation of human immunodeficiency virus type 1 from semen and vaginal fluids. Methods Mol Biol 2005;304:71-86.

3. Ball J, Curran R, Irving W, Dearden A. HIV1 in semen: determination of proviral and viral titres compared to blood, and quantification of semen leukocyte populations. J Med Virol 1999;59:356-63.

4. Suresh P, Wanchu A. Chemokines and chemokine receptors in HIV infection: role in pathogenesis and therapeutics. J Postgrad Med 2006;52:210-7.

5. Eslahpazir J, Jenabian M, Bouhlal $\mathrm{H}$, et al. Infection of macrophages and dendritic cells with primary R5-tropic human immunodeficiency virus type 1 inhibited by natural polyreactive anti-CCR5 antibodies purified from cervicovaginal secretions. Clin Vaccine Immunol 2008;15:87284.

6. Naarding M, Ludwig I, Groot F, et al. Lewis $\mathrm{X}$ component in human milk binds DCSIGN and inhibits HIV-1 transfer to CD4+ T lymphocytes. J Clin Invest 2005;115: 3256-64.

7. Sabatté J, Ceballos A, Raiden S, et al. Human seminal plasma abrogates the capture and transmission of human immunodeficiency virus type 1 to CD4+ T cells mediated by DC-SIGN. J Virol 2007;81: 13723-34.

8. Ghezzi S, Menzo S, Brambilla A, et al. Inhibition of R5X4 dualtropic HIV-1 primary isolates by single chemokine coreceptor ligands. Virology 2001;280:253-61.

9. De Clercq E, Schols D. Inhibition of HIV infection by CXCR4 and CCR5 chemokine receptor antagonists. Antivir Chem Chemother 2001;12 Suppl 1:19-31.

10. Feng Z, Dubyak G, Lederman M, Weinberg A. Cutting edge: human beta defensin 3-a novel antagonist of the HIV-1 coreceptor CXCR4. J Immunol 2006;77:782-6.

11. Gilliam BL RD, Redfield RR. Clinical use of CCR5 inhibitors in HIV and beyond. J Transl Med 2011;9 Suppl 1:S9.

12. Telwatte S, Moore K, Johnson A, et al. Virucidal activity of the dendrimer microbicide SPL7013 against HIV-1. Antiviral Res 2011;90:195-99.

13. Whaley K, Hanes J, Shattock R, et al. Novel approaches to vaginal delivery and safety of microbicides: biopharmaceuticals, nanoparticles, and vaccines. Antiviral Res 2010;88 Suppl 1:S55-66.

14. Pérez-Anes A, Stefaniu C, Moog C, et al. Multivalent catanionic GalCer analogs derived from first generation dendrimeric phosphonic acids. Bioorg Med Chem 2010;18:242-8.

15. Ham A, Cost M, Sassi A, et al. Targeted delivery of PSC-RANTES for HIV-1 prevention using biodegradable nanoparticles. Pharm Res 2009;26:502-11.

16. Trecarichi EMTM, de Gaetano Donati K, Tamburrini E, et al. Partial protective effect of CCR5-Delta 32 heterozygosity in a cohort of heterosexual Italian HIV-1 exposed uninfected individuals. AIDS Res Ther 2006;25:22.

17. Philpott SWB, Tarwater P, Vermund SH, et al. CC chemokine receptor 5 genotype and susceptibility to transmission of human immunodeficiency virus type 1 in women. J Infect Dis 2003;187:569-75.

18. Kaiser P, Symons R, Shah S, et al. RNAibased treatment for neovascular age-related macular degeneration by sirna-027. Am J Ophthalmol 2010;150:33-9.

19. Czech MPAM, Tesz GJ. RNAi-based thera- peutic strategies for metabolic disease. Nat Rev Endocrinol 2011;epub ahead of print.

20. Di Paolo DBC, Pastorino F, Carosio R, et al. Neuroblastoma-targeted Nanoparticles Entrapping siRNA Specifically Knockdown ALK. Mol Ther 2011; epub ahead of print.

21. Zhou J, Rossi J. Progress in RNAi-based antiviral therapeutics. Methods Mol Biol 2011;721:65-75.

22. Hobel S, Prinz RAM, Urban-Klein B, et al. Polyethylenimine PEI F25-LMW allows the long-term storage of frozen complexes as fully active reagents in siRNA-mediated gene targeting and DNA delivery. Eur $\mathrm{J}$ Pharma Biopharma 2008;70:29-41.

23. Lee N, Dohjima T, Bauer G, et al. Expression of small interfering RNAs targeted against HIV-1 rev transcripts in human cells. Nat Biotechnol 2002;20:5005.

24. Das A, Brummelkamp T, Westerhout E, et al. Human immunodeficiency virus type 1 escapes from RNA interference-mediated inhibition. J Virol 2004;78:2601-5.

25. Boden D, Pusch 0, Silbermann R, et al. Enhanced gene silencing of HIV-1 specific siRNA using microRNA designed hairpins. Nucleic Acids Res 2004;32:1154-8.

26. Martínez M, Gutiérrez A, Armand-Ugón M, et al. Suppression of chemokine receptor expression by RNA interference allows for inhibition of HIV-1 replication. AIDS 2002;16:2385-90.

27. Arrighi J, Pion M, Wiznerowicz M, et al. Lentivirus-mediated RNA interference of DC-SIGN expression inhibits human immunodeficiency virus transmission from dendritic cells to $\mathrm{T}$ cells. $\mathrm{J}$ Virol 2004;78:10848-55.

28. Kim SS, Peer D, Kumar P, et al. RNAimediated CCR5 silencing by LFA-1 targeted nanoparticles prevents HIV infection in BLT mice. Mol Therapy 2010;18:370-6.

29. Kumar P, Ban HS, Kim SS, et al. T Cell-specific siRNA delivery suppresses HIV-1 infection in humanized mice. Cell 2008;134:577-86.

30. Mallipeddi R, Rohan L. Nanoparticle-based vaginal drug delivery systems for HIV prevention. Expert Opin Drug Del 2010;7:3748.

31. Rohan L, Sassi A. Vaginal drug delivery systems for HIV prevention. AAPS J 2009;11:78-87.

32. Asin S, Eszterhas S, Rollenhagen C, et al. HIV-1 infection in women: Increased transcription in ectocervical tissue explants. J Infect Dis 2009;200:965-72.

33. Cubillos-Ruiz J, Fiering S, Conejo-Garcia J. Nanomolecular targeting of dendritic cells for ovarian cancer therapy. Future Oncol 2009:5:1189-92.

34. Mehta N, Trzmielina S, Nonvane B, et al. 
Low-cost HIV-1 diagnosis and quantification in dried blood spots by real time PCR. PLoS ONE 2009;4:e5819.

35. Lyimo M, Howell A, Balandya E, et al. Innate factors in human breast milk inhibit cell-free HIV-1 but not cell-associated HIV-1 Infection of CD4+ cells. J Acquir Immune Defic Syndr 2009;5:117-24.

36. Peng W, Henderson G, Inman M, et al. The locus encompassing the latency-associated transcript of herpes simplex virus type 1 interferes with and delays interferon expression in productively infected neuroblastoma cells and tigeminal ganglia of acutely infected mice. J Virol 2005;79:6162-71.

37. Anderson D, Pudney J, Schust D. Caveats associated with the use of human cervical tissue for HIV and microbicide research. AIDS 2010;24:1-4.

38. Thubagere ARB. Nanoparticle-induced apoptosis propagates through hydrogenperoxide-mediated bystander killing: insights from a human intestinal epithelium in vitro model. ACS Nano 2010;4:361122.

39. Hock SCYY, Wah CL. A review of the current scientific and regulatory status of nanomedicines and the challenges ahead. PDA J Pharm Sci Technol 2011;65:177-95.

40. Youns MHJ, Efferth T. Therapeutic and diagnostic applications of nanoparticles. Curr Drug Targets 2011;12:357-65.

41. Yeaman G, Asin S, Weldon S, et al. Chemokine receptor expression in the human ectocervix: implications for infec- tion by the human immunodeficiency virus-type I. Immunology 2004;113:524-33.

42. Yeaman G, Howell A, Weldon S, et al. Human immunodeficiency virus receptor and coreceptor expression on human uterine epithelial cells: regulation of expression during the menstrual cycle and implications for human immunodeficiency virus infection. Immunology 2003;109:13746.

43. Yang K, Puel A, Zhang S, et al. Human TLR-7, -8-, and -9-mediated induction of IFN-alpha/beta and -lambda is IRAK-4 dependent and redundant for protective immunity to viruses. Immunity 2005;23: 465-78.

44. Bourquin CWC, Heidegger S, Fuchs S, et al. Delivery of immunostimulatory RNA oligonucleotides by gelatin nanoparticles triggers an efficient antitumoral response. J Immunother 2010;33:935-44.

45. Chen H, Sun B, Tran K, Shen H. Effects of particle size on toll-like receptor 9-mediated cytokine profiles. Biomaterials 2011;32:1731-7.

46. Kerkmann MLD, Weyermann J, Marschner A, et al. Immunostimulatory properties of CpG-oligonucleotides are enhanced by the use of protamine nanoparticles. Oligonucleotides 2006;16:313-22.

47. Cubillos-Ruiz J, Engle X, Scarlett U, et al. Polyethylenimine-based siRNA nanocomplexes reprogram tumor-associated dendritic cells via TLR5 to elicit therapeutic antitumor immunity. J Clin Invest 2009; 119:2231-44.
48. O'Brien MMO, Sabado RL, Baranda SJ, et al. Spatiotemporal trafficking of HIV in human plasmacytoid dendritic cells defines a persistently IFN- $\alpha$-producing and partially matured phenotype. J Clin Invest 2011;121:1088-101.

49. Goujon C, Malim M. Characterization of the alpha interferon-induced postentry block to HIV-1 infection in primary human macrophages and T cells. J Virol 2010;84: 9254-66.

50. Lara H, Ixtepan-Turrent L, Garza-Treviño E, Rodriguez-Padilla C. PVP-coated silver nanoparticles block the transmission of cell-free and cell-associated HIV-1 in human cervical culture. J Nanobiotechnol 2010;8:1-11.

51. das Neves J, Amiji M, Sarmento B. Mucoadhesive nanosystems for vaginal microbicide development: friend or foe? Wiley Interdiscip Rev Nanomed Nanobiotechnol 2011; epub ahead of print.

52. Alukda D, Strugis T, Youan B. Formulation of tenofovir-loaded functionalized solid lipid nanoparticles intended for HIV prevention. J Pharm Sci 2011;epub ahead of print.

53. Brache V, Faundes A. Contraceptive vaginal rings: A review. Contraception 2010;82: 418-27.

54. Johnson TJ GK, Fabian J, Albright TH, Kiser PF. Segmented polyurethane intravaginal rings for the sustained combined delivery of antiretroviral agents dapivirine and tenofovir. Eur J Pharma Sci 2010;39: 203-12. 